\title{
ON POLYGONS IN REAL PROJECTIVE $n$-SPACE
}

\author{
DOUGLAS DERRY
}

Although Juel [2] made full use of the differentiability assumption in the proofs used in his theory of differentiable curves of bounded real order, many of the results seem to be true if the differentiability is weakened. This has already been pointed out by Hjelmslev (cf. [1], where polygons in the projective plane and polyhedra in the projective space are considered). The present paper develops a theory of polygons analogous to the simplest of Juel's curves, those of order $n$ in $n$-space. The methods are related to those used by Kivikoski [3] [4] in his study of polygons in the projective plane. The principal result, given in 4.6 , is that each such polygon may be obtained from a polygon connecting the vertices of a simplex by a succession of inscribed polygons defined in 3.1. Two applications of the results are given. The first of these, in 5.6, is a proof of a duality theorem for these polygons analogous to the one proved by Scherk [6] for differentiable curves. The second in 6.1, related to the first, proves a result for continuous but not necessarily differentiable curves. This result for $n=3$ follows from a result of Marchaud [5] on which he based his analysis of continuous plane curves of real order 3 .

\section{Definitions and notation.}

A polygon $\pi$ in real projective $n$-space is defined by its sides $A_{1} A_{2}$, $A_{2} A_{3}, \ldots, A_{r-1} A_{r}$ and in the case in which it is closed $A_{r} A_{1}$. The consecutive endpoints $A_{1}, A_{2}, \ldots, A_{r}$ of the sides are the vertices of $\pi$. If the polygon is closed it is useful to define the vertex subscripts $i$ modulo $r$. Thus $A_{1}, A_{1+r}$ both represent the same vertex.

A point common to a linear $k$-space $L, 0 \leqq k \leqq n-1$, and a polygon $\pi$ is defined to be an intersection point of $L$ and $\pi$ if it is a vertex of $\pi$ or if it is the only point of a side of $\pi$ which is within $L$.

A polygon $\pi_{n}$ with vertices $A_{1}, A_{2}, \ldots, A_{r}$ is defined to be a polygon of order $n$ in $n$-space provided it satisfies

Received April 18, 1958. 
(1) the dimension condition that it is not contained in a hyperplane, and

(2) the order condition that no hyperplane intersect $\pi_{n}$ in more than $n$ points.

The symbol $\pi_{n}$ will be used exclusively for polygons which satisfy these two conditions.

If $Q_{1}, Q_{2}, \ldots, Q_{r}$ are points in real projective $n$-space then the linear space generated by these points will be designated by the symbol $\left[Q_{1}, Q_{2}, \ldots, Q_{r}\right]$.

\section{Preliminary lemmas.}

2.1. If $B_{0}, B_{1}, \ldots, B_{k}, 0 \leqq k \leqq n$, are vertices of a polygon $\pi_{n}$ then $\left[B_{0}, B_{1}, \ldots, B_{k}\right]$ has dimension $k$.

Proof. If $\left[B_{0}, B_{1}, \ldots, B_{k}\right]$ had dimension less than $k$ then, as $\pi_{n}$ satisfies the dimension condition, vertices $B_{k+1}, B_{k+2}, \ldots, B_{m}, m>n$, would exist so that $\left[B_{0}, B_{1}, \ldots, B_{m}\right]$ had dimension $n-1$. As this would contradict the order condition, 2.1 is proved.

This result implies that the vertices of a polygon $\pi_{n}$ must be distinct.

2.2. If, for $n \geqq 2, \dot{A}_{j}, \dot{A}_{j-1} \dot{A}_{j}$ be the projections of the vertex $A_{j}$ and the side $A_{j-1} A_{j}, i \neq j, i \neq j-1$, of a closed polygon $\pi_{n}$ from a vertex $A_{i}$ onto a hyperplane, then the projection of $\pi_{n}$ from $A_{i}$ is an open polygon $\pi_{n-1}$ with sides $\dot{A}_{1} \dot{A}_{2}, \ldots, \dot{A}_{i-2} \dot{A}_{i-1}, \dot{A}_{i+1} \dot{A}_{i+2}, \ldots, \dot{A}_{r} \dot{A}_{1}$.

Where $\dot{A}_{i-1} \dot{A}_{i+1}$ is defined to be the projection of the third side of the odd triangle with sides $A_{i-1} A_{i}, A_{i} A_{i+1}$ then $\pi_{n-1}$ may be closed by the addition of $\dot{A}_{i-1} \dot{A}_{i+1}$ without increase of order.

Proof. If $A_{i} \in\left[A_{j-1}, A_{j}\right]$ then by 2.1 either $i=j$ or $i=j-1$. Hence the projection of the side $A_{j-1} A_{j}$ of $\pi_{n}$ from the vertex $A_{i}$ is a line segment $\dot{A}_{j-1} \dot{A}_{j}$ if $i \neq j, i \neq j-1$. Let $A_{i-1} A_{i+1}$ be the third side of the odd triangle $t$ which contains the two sides $A_{i-1} A_{i}, A_{i} A_{i+1}$. Where $\dot{A}_{i-1} \dot{A}_{i+1}$ is defined as the projection of $A_{i-1} A_{i+1}$ from the vertex $A_{i}$, let $\pi$ be the closed polygon which consists of the segments $\dot{A}_{1} \dot{A}_{2}, \ldots, \dot{A}_{i-2} \dot{A}_{i-1}, \dot{A}_{i-1} \dot{A}_{i+1}$, $\dot{A}_{i+1} \dot{A}_{i+2}, \ldots, \dot{A}_{r} \dot{A}_{1} . \pi$ is not included in a hyperplane of the projected space, for otherwise $\pi_{n}$ would be included in a hyperplane of the original space.

To complete the proof it is sufficient to show $\pi$ has order $n-1$. To do this let $\dot{H}$ be any hyperplane of the projected space and $H$ the hyperplane of the original space, the projection of which from $A_{i}$ is $\dot{H}$. Suppose first that $H$ contains no interior point of $A_{i-1} A_{i+1}$. In this case each intersection of $\dot{H}$ and $\pi$ is the projection of an intersection point of $H$ 
and $\pi_{n}$ different from $A_{i}$. As $\pi_{n}$ has order $n$ and $A_{i}$ is an intersection point there are at most $n-1$ such intersection points. Hence $\dot{H}$ intersects $\pi$ in at most $n-1$ points. We now consider the case in which $H$ does contain an interior point of $A_{i-1} A_{i+1}$. $H$ now supports the triangle $t$ at $A_{i}$ as $t$ is odd. Let $L$ be the linear subspace generated by the vertices of $\pi_{n}$ within $H$ which are different from $A_{i}$. It follows from 2.1 that $A_{i} \notin L$. Hence $H$ may be subjected to a displacement to a position $\bar{H}$ so that the points of $L$ remain fixed but so that $\ddot{H}$ intersects $A_{i-1} A_{i}$ in an interior point. If this displacement of $H$ is sufficiently small the original points of intersection of $H$ and $\pi_{n}$ will each move in arbitrarily small neighborhoods. As $H$ supports $t$ at $A_{i}, \bar{H}$ will also intersect $A_{i} A_{i+1}$ in an interior point. Thus $\bar{H}$ contains exactly one more intersection of $\pi_{n}$ than $H$. Therefore $H$ intersects $\pi_{n}$ in at most $n-1$ points including $A_{i}$. The hyperplane $\dot{H}$ intersects $\dot{A}_{i-1} \dot{A}_{i+1}$ in an interior point as $H$ is assumed to intersect $A_{i-1} A_{i+1}$ in an interior point. All the other intersection points of $\dot{H}$ and $\pi$ are projections of intersection points of $H$ and $\pi_{n}$ different from $A_{i}$. As we have proved there are at most $n-2$ such points it follows that $\dot{H}$ intersects $\pi$ in at most $n-1$ points. Thus $\pi$ has order $n-1$. The proof is now complete.

2.3. If $\sigma_{n}$ is a closed polygon not within a hyperplane with exactly $n+1$ sides and if a hyperplane exists which intersects $\sigma_{n}$ in exactly $n$ points each of which is interior to a side, then $\sigma_{n}$ has order $n$.

Proof. We first show that a hyperplane which does not contain vertices of $\sigma_{n}$ intersects $\sigma_{n}$ in at most $n$ points. By the hypothesis a hyperplane $H$ exists which intersects $\sigma_{n}$ in exactly $n$ points each of which is an interior point of a side. Hence successive vertices $A_{1}, A_{2}, \ldots, A_{n+1}$ may be chosen so that $H$ does not intersect the side $A_{n+1} A_{1}$. If $P_{i}$ be the intersection point of $H$ and the side $A_{i} A_{i+1}, 1 \leqq i \leqq n$, then $\left[P_{1}, P_{2}, \ldots, P_{n}\right]$ is a hyperplane. Otherwise $\left[A_{1}, P_{1}, \ldots, P_{n}\right]$ would have dimension at most $n-1$ and contain all the vertices of $\sigma_{n}$ as $P_{1}, P_{2}, \ldots, P_{n}$ are interior points of consecutive sides. Hence $\sigma_{n} \subseteq\left[A_{1}, P_{1}, \ldots, P_{n}\right]$, contrary to the hypothesis that $\sigma_{n}$ is not in a hyperplane. This also proves that if $P_{i}$ varies continuously but remains an interior point of $A_{i} A_{i+1}, 1 \leqq i \leqq n$, then $\left[P_{1}, P_{2}, \ldots, P_{n}\right]$ is a hyperplane which varies continuously and can never enter a vertex of $\sigma_{n}$. Hence no position of this hyperplane can intersect the side $A_{n+1} A_{1}$. Consequently no hyperplane can intersect $\sigma_{n}$ in $n+1$ points, none of which are vertices as such a hyperplane would be one of the hyperplanes $\left[P_{1}, P_{2}, \ldots, P_{n}\right]$.

Now let $H$ be a hyperplane which intersects $\sigma_{n}$ in at least one vertex. As $\sigma_{n}$ is not contained in a hyperplane, not all of the vertices can be con- 
tained in $H$. Hence $H$ contains at least one vertex, say $A_{1}$, so that a consecutive vertex, say $A_{2}$, is not contained in $H$. This means that $H$ contains only the point $A_{1}$ of the line $\left[A_{1}, A_{2}\right] . A_{1}$ cannot be within the space generated by the other vertices of $\sigma_{n}$ within $H$ for then all vertices of $\sigma_{n}$ would be within a hyperplane. Hence $H$ may be displaced so that its displaced position $\bar{H}$ intersects the line $\left[A_{1}, A_{2}\right]$ in an interior point $A$ while each vertex of $\sigma_{n}$ within $H$ other than $A_{1}$ remains within $\bar{H}$. If the displacement is sufficiently small any intersection point of $H$ and $\sigma_{n}$ is displaced into an intersection point of $\bar{H}$ and $\sigma_{n}$. This process may be repeated until a hyperplane is obtained which intersects $\sigma_{n}$ only in interior points of its sides and which does not intersect $\sigma_{n}$ in fewer points than $H$. As such a hyperplane intersects $\sigma_{n}$ in at most $n$ points by the result of the first paragraph, $H$ intersects $\sigma_{n}$ in at most $n$ points. Hence $\sigma_{n}$ has order $n$ and the proof is complete.

\section{The inscribed polygons $I\left(\pi_{n}\right)$.}

3.1 Definition. If $n$ consecutive sides $A_{1} A_{2}, A_{2} A_{3}, \ldots, A_{n} A_{n+1}$ of $a$ closed polygon $\pi_{n}$ uith vertices $A_{i}, 1 \leqq i \leqq r$, are each subdivided into segments $A_{i} B_{i+1}, B_{i+1} A_{i+1}$ by an interior point $B_{i+1}$ and if $B_{i} B_{i+1}$ is defined so that $B_{i} B_{i+1}, B_{i} A_{i}, A_{i} B_{i+1}$ is an even triangle, then $I\left(\pi_{n}\right)$ is defined to be the polygon inscribed in $\pi_{n}$ with sides $A_{1} B_{2}, B_{2} B_{3}, \ldots, B_{n} B_{n+1}, B_{n+1} A_{n+1}$, $A_{n+1} A_{n+2}, \ldots, A_{r} A_{1}$.

3.2 The polygons $I\left(\pi_{n}\right)$ satisfy the dimension condition and have order $n$.

Proof. As the vertices of $\pi_{n}$ are linear combinations of the vertices of $I\left(\pi_{n}\right)$ it follows that if $I\left(\pi_{n}\right)$ is included in a hyperplane then this hyperplane also contains $\pi_{n}$. Hence, as $\pi_{n}$ satisfies the dimension condition, $I\left(\pi_{n}\right)$ also satisfies this condition.

Let $H$ be a given hyperplane. We define a one to one correspondence between the intersection points of $H$ and $I\left(\pi_{n}\right)$ and intersection points of $H$ and $\pi_{n}$. Let $Q$ be an intersection point of $H$ and $I\left(\pi_{n}\right)$. Suppose first that $Q$ is a vertex $B_{i}$ of $I\left(\pi_{n}\right)$ which is not an intersection point of $H$ and $\pi_{n}$. This means that $B_{i}$ is an interior point of a side $A_{i-1} A_{i}$ of $\pi_{n}$ which is part of a polygon $\operatorname{arc} A_{m} A_{m+1} \ldots A_{m+h}, 1 \leqq m, m+h \leqq n+1$, entirely within $H$. This polygon arc may be assumed to have maximum length. $m=1$ and $m+h=n+1$ cannot be true simultaneously for otherwise $H$ would contain $n+1$ vertices of $\pi_{n}$. If $m+h=n+1$ then $m \neq 1$ and we define $\bar{Q}$ to be $A_{i-1}$. If $m+h<n+1, \bar{Q}$ is defined to be $A_{i}$. In each case $\bar{Q}$ is a vertex of $\pi_{n}$ within $H$ which is not a point of $I\left(\pi_{n}\right)$. Now suppose $Q$ is an interior point of a side $B_{i} B_{i+1}, 2 \leqq i \leqq n$. In this case $H$ contains exactly 
one more point of the even triangle with sides $B_{i} B_{i+1}, B_{i} A_{i}, A_{i} B_{i+1}$. We define this additional point to be $\bar{Q}$. $\bar{Q}$ must be on either $B_{i} A_{i}$ or $A_{i} B_{i+1}$. As $B_{i}, B_{i+1} \notin H, \bar{Q}$ is a point of intersection of $H$ and $\pi_{n} . \bar{Q}$ may be the vertex $A_{i}$ but in this case neither side $A_{i-1} A_{i}$ nor $A_{i} A_{i+1}$ can be within $H$. Finally, any additional intersection point $Q$ of $H$ and $I\left(\pi_{n}\right)$ is also an intersection point of $H$ and $\pi_{u}$ and so in this case we define $\bar{Q}=Q$. As the correspondence $Q \rightarrow \bar{Q}$ is a one to one correspondence, $H$ cannot intersect $I\left(\pi_{n}\right)$ in a greater number of points than it intersects $\pi_{n}$. Hence $I\left(\pi_{n}\right)$ has order $n$ and the result is proved.

\section{The escribed polygons $E_{k}\left(\pi_{n}\right)$.}

In this section it is shown that every polygon $\pi_{n}$ is either a polygon with $n+1$ vertices or can be obtained from such a polygon by constructing a succession of inscribed polygons.

4.1 Definition of $B_{q}^{k}$. If, for $r>n+1, A_{1}, A_{2}, \ldots, A_{r}$ are the vertices of a closed polygon $\pi_{n}$, then, for $1 \leqq q \leqq n+1, B_{q}^{0}$ is defined to be the vertex $A_{q}$ and $B_{q}^{k}$ the intersection

$$
\left[A_{1}, A_{2}, \ldots, A_{q}\right] \cap\left[A_{k+q}, A_{k+q+1}, \ldots, A_{k+n+1}\right],
$$

$1 \leqq k \leqq r-n-1$.

4.2 (1) $B_{1}^{k}=A_{1}, B_{n+1}^{k}=A_{k+n+1}$, (2) $B_{q}^{k}$ is a point and (3) $\left[B_{q}^{k}, B_{q+1}^{k}\right]$ is a line which contains $B_{q+1}^{k-1}, B_{q+1}^{k-1} \neq B_{q}^{k}, B_{q+1}^{k-1} \neq B_{q+1}^{k}, 1 \leqq k \leqq r-n-1,1 \leqq q \leqq n$.

Proof. As $\pi_{n}$ has order $n$ any $n+1$ distinct vertices of $\pi_{n}$ generate the full projective $n$-space. It follows, then, from the definitions

$$
\begin{aligned}
& B_{1}^{k}=A_{1} \cap\left[A_{k+1}, A_{k+2}, \ldots, A_{k+n+1}\right], \\
& B_{n+1}^{k}=\left[A_{1}, A_{2}, \ldots, A_{n+1}\right] \cap A_{k+n+1},
\end{aligned}
$$

that $B_{1}^{k}=A_{1}$ and $B_{n+1}^{k}=A_{k+n+1}$. Thus (1) is proved.

The spaces $\left[A_{1}, A_{2}, \ldots, A_{q}\right],\left[A_{k+q}, A_{k+q+1}, \ldots, A_{k+n+1}\right]$, and

$$
\left[A_{1}, A_{2}, \ldots, A_{q}, A_{k+q}, \ldots, A_{k+n+1}\right]
$$

contain $q, n+2-q$, and $n+2$ distinct vertices of $\pi_{n}$, respectively, as $k \leqq r-n-1$. It follows, then, from 2.1 that these spaces have dimension $q-1, n+1-q$, and $n$ respectively. If $d$ is the dimension of the intersection

$$
\left[A_{1}, A_{2}, \ldots, A_{q}\right] \cap\left[A_{k+q}, A_{k+q+1}, \ldots, A_{k+n+1}\right]=B_{q}^{k}
$$

it follows from the incidence relation $(q-1)+(n+1-q)=n+d$ that $d=0$. Hence $B_{q}^{k}, 1 \leqq k \leqq r-n-1,1 \leqq q \leqq n+1$, is a point and (2) is proved. 
It follows from 4.1 that $B_{q}^{k} \in\left[A_{1}, A_{2}, \ldots, A_{q}\right]$ and

$$
B_{q+1}^{k} \in\left[A_{k+q+1}, A_{k+q+2}, \ldots, A_{k+n+1}\right] .
$$

By the method used in the previous paragraph the intersection

$$
\left[A_{1}, A_{2}, \ldots, A_{q}\right] \cap\left[A_{k+q+1}, A_{k+q+2}, \ldots, A_{k+n+1}\right], \quad 1 \leqq q \leqq n,
$$

has dimension -1 and so is empty. Therefore $B_{q}^{k} \neq B_{q+1}^{k}$ and consequently $\left[B_{q}^{k}, B_{q+1}^{k}\right]$ is a straight line, $1 \leqq k \leqq r-n-1,1 \leqq q \leqq n$. It follows from 4.1 that

$$
\left[B_{q}^{k}, B_{q+1}^{k}\right] \subseteq\left[A_{1}, A_{2}, \ldots, A_{q+1}\right] \cap\left[A_{k+q}, A_{k+q+1}, \ldots, A_{k+n+1}\right] .
$$

This intersection has dimension 1 by the method of the previous paragraph. Hence

$$
\left[B_{q}^{k}, B_{q+1}^{k}\right]=\left[A_{1}, A_{2}, \ldots, A_{q+1}\right] \cap\left[A_{k+q}, A_{k+q+1}, \ldots, A_{k+n+1}\right],
$$

$1 \leqq k \leqq r-n-1,1 \leqq q \leqq n$. Consequently, for $k=1$,

$$
B_{q+1}^{k-1}=B_{q+1}^{0}=A_{q+1} \in\left[B_{q}^{k}, B_{q+1}^{k}\right] .
$$

If $k>1, B_{q+1}^{k-1}$ is defined to be

$$
B_{q+1}^{k-1}=\left[A_{1}, A_{2}, \ldots, A_{q+1}\right] \cap\left[A_{k+q}, A_{k+q+1}, \ldots, A_{k+n}\right]
$$

which point is likewise contained in $\left[B_{q}^{k}, B_{q+1}^{k}\right]$. Thus

$$
B_{q+1}^{k-1} \in\left[B_{q}^{k}, B_{q+1}^{k}\right], \quad 1 \leqq k \leqq n-r-1, \quad 1 \leqq q \leqq n .
$$

It remains to prove

Suppose for $k>1$,

$$
B_{q+1}^{k-1} \neq B_{q}^{k}, \quad B_{q+1}^{k-1} \neq B_{q+1}^{k} .
$$

$$
\begin{aligned}
B_{q+1}^{k-1} & =\left[A_{1}, A_{2}, \ldots, A_{q+1}\right] \cap\left[A_{k+q}, A_{k+q+1}, \ldots, A_{k+n}\right] \\
& =\left[A_{1}, A_{2}, \ldots, A_{q}\right] \cap\left[A_{k+q}, A_{k+q+1}, \ldots, A_{k+n+1}\right]=B_{q}^{k} .
\end{aligned}
$$

Then the intersection $\left[A_{1}, A_{2}, \ldots, A_{q}\right] \cap\left[A_{k+q}, A_{k+q+1}, \ldots, A_{k+n}\right]$ cannot be empty as it contains $B_{q}^{k}$. However, this intersection is the intersection of two spaces of dimension $q-1$ and $n-q$ which together generate the whole space. If $d$ be its dimension it follows from the incidence relation $(q-1)+(n-q)=n-d$ that $d=-1$ which means the intersection is empty. This contradiction proves $B_{q+1}^{k-1} \neq B_{q}^{k}, k>1$. We now suppose, for $k>1$, that

$$
\begin{aligned}
B_{q+1}^{k-1} & =\left[A_{1}, A_{2}, \ldots, A_{q+1}\right] \cap\left[A_{k+q}, A_{k+q+1}, \ldots, A_{k+n}\right] \\
& =\left[A_{1}, A_{2}, \ldots, A_{q+1}\right] \cap\left[A_{k+q+1}, A_{k+q+2}, \ldots, A_{k+n+1}\right]=B_{q+1}^{k} .
\end{aligned}
$$

It follows from 2.1 that 
Hence

$$
\left[A_{k+q}, A_{k+q+1}, \ldots, A_{k+n}\right] \cap\left[A_{k+q+1}, A_{k+q+2}, \ldots, A_{k+n+1}\right]
$$

$$
=\left[A_{k+q+1}, A_{k+q+2}, \ldots, A_{k+n}\right] .
$$

$$
B_{q+1}^{k-1}=B_{q+1}^{k} \in\left[A_{1}, A_{2}, \ldots, A_{q+1}\right] \cap\left[A_{k+q+1}, A_{k+q+2}, \ldots, A_{k+n}\right] .
$$

This intersection is the intersection of two spaces of dimension $q$ and $n-q-1$ which together generate the whole space. As before it follows from the incidence relation that it is empty and so $B_{q+1}^{k-1} \neq B_{q+1}^{k}$. Finally let $k=1$. In this case $B_{q+1}^{0}=A_{q+1}=B_{q}^{1}$ would imply $A_{q+1} \in\left[A_{1}, A_{2}, \ldots, A_{q}\right]$ which is impossible because of 2.1. Likewise $B_{q+1}^{0}=B_{q+1}^{1}$ would imply $A_{q+1} \in\left[A_{2+q}, A_{3+q}, \ldots, A_{n+k+1}\right]$ which is also impossible because of 2.1 . This completes the proof.

These results enable us to introduce

4.3 Definition of $E_{k}\left(\pi_{n}\right)$. If $A_{1}, A_{2}, \ldots, A_{r}, r>n+1$, are the vertices of a closed polygon $\pi_{n}$ and $B_{q}^{k}, 1 \leqq k \leqq r-n-1,1 \leqq q \leqq n+1$, are defined in 4.1 then $B_{q}^{k} B_{q+1}^{k}, 1 \leqq q \leqq n$, is defined to be the line segment in $\left[B_{q}^{k}, B_{q+1}^{k}\right]$ which contains $B_{q+1}^{k-1}$.

$E_{k}\left(\pi_{n}\right)$ is defined to be $\pi_{n}$ if $k=0$ and the polygon with sides $B_{1}^{k} B_{2}^{k}$, $B_{2}^{k} B_{3}^{k}, \ldots, B_{n}^{k} B_{n+1}^{k}\left(=B_{n}^{k} A_{n+k+1}\right), \quad A_{n+k+1} A_{n+k+2}, \ldots, A_{r} A_{1}\left(=A_{r} B_{1}^{k}\right) \quad$ if $1 \leqq k \leqq r-n-1$.

4.4 If, for $1 \leqq k \leqq r-n-1$ and $n \geqq 2, t_{i}^{k}, 2 \leqq i \leqq n$, is the triangle consisting of the side $B_{i}^{k-1} B_{i+1}^{k-1}$ of $E_{k-1}\left(\pi_{n}\right)$ and the segments $B_{i}^{k-1} B_{i}^{k}, B_{i}^{k} B_{i+1}^{k-1}$ of the sides $B_{i-1}^{k} B_{i}^{k}, B_{i}^{k} B_{i+1}^{k}$ of $E_{k}\left(\pi_{n}\right)$, then $t_{i}^{k} i$ an even triangle.

Proof. Let $A_{1}, A_{2}, \ldots, A_{r}$ be the vertices of the closed polygon $\pi_{n}$. We first consider the projection of $E_{k}\left(\pi_{n}\right)$ from the vertex $A_{1}$ for $n \geqq 2$. If $\dot{A}_{i-1}$ be the projection of $A_{i}$ from $A_{1}, i \neq 1$, then the $r-1$ points $\dot{A}_{1}, \dot{A}_{2}, \ldots, \dot{A}_{r-1}$ are the vertices of a closed polygon $\pi_{n-1}$. Each side $\dot{A}_{i} \dot{A}_{i+1}, 1 \leqq i \leqq r-2$, of $\pi_{n-1}$ is the projection of the side $A_{i+1} A_{i+2}$ of $\pi_{n}$ while the side $\dot{A}_{r-1} \dot{A}_{1}$ is defined as in 2.2. If $\dot{B}_{q}^{k}$ is defined to be

$$
\dot{B}_{q}^{k}=\left[\dot{A}_{1}, \dot{A}_{2}, \ldots, \dot{A}_{q}\right] \cap\left[\dot{A}_{k+q}, \dot{A}_{k+q+1}, \ldots, \dot{A}_{n+k}\right],
$$

$1 \leqq k \leqq(r-1)-(n-1)-1=r-n-1,1 \leqq q \leqq n$, then $\dot{B}_{1}^{k}, \dot{B}_{2}^{k}, \ldots, \dot{B}_{n}^{k}, \dot{A}_{n+k}$, $\ldots, \dot{A}_{r-1}$ are the vertices of $E_{k}\left(\pi_{n-1}\right)$ in accordance with 4.3. It follows from the definition of $B_{q}^{k}$ in 4.1 that its projection from $A_{1}$ is $\dot{B}_{q-1}^{k}$, $2 \leqq q \leqq n+1$. As $B_{1}^{k}=A_{1}$, the side $B_{1}^{k} B_{2}^{k}$ is projected into the single point $\dot{B}_{1}^{k}$. To consider the sides $B_{q}^{k} B_{q+1}^{k}, 2 \leqq q \leqq n$, we note that, for $1<k \leqq r-n-1$, the interior point

$$
B_{q+1}^{k-1}=\left[A_{1}, A_{2}, \ldots, A_{q+1}\right] \cap\left[A_{k+q}, A_{k+q+1}, \ldots, A_{n+k}\right]
$$

of $B_{q}^{k} B_{q+1}^{k}$ is projected into the interior point 


$$
\dot{B}_{q}^{k-1}=\left[\dot{A}_{1}, \dot{A}_{2}, \ldots, \dot{A}_{q}\right] \cap\left[\dot{A}_{k+q-1}, \dot{A}_{k+q}, \ldots, \dot{A}_{k+n-1}\right]
$$

of $\dot{B}_{q-1}^{k} \dot{B}_{q}^{k}$ while for $k=1$ the interior point $B_{q+1}^{0}=A_{q+1}$ of $B_{q}^{1} B_{q+1}^{1}$ is projected into the interior point $\dot{B}_{q}^{0}=\dot{A}_{q}$ of $\dot{B}_{q-1}^{1} \dot{B}_{q}^{1}$. Thus $B_{q}^{k} B_{q+1}^{k}$ is projected into the line segment with endpoints $\dot{B}_{q-1}^{k}, \dot{B}_{q}^{k}$ which contains the point $\dot{B}_{q}^{k-1}$ in its interior which is, by the definition, the side $\dot{B}_{q-1}^{k} \dot{B}_{q}^{k}$ of $E_{k}\left(\pi_{n-1}\right), 2 \leqq q \leqq n, 1 \leqq k \leqq n-r-1$. Each side $A_{i} A_{i+1}, n+k+1 \leqq i \leqq r-1$, is projected into the side $\dot{A}_{i-1} \dot{A}_{i}$ of $E_{k}\left(\pi_{n-1}\right)$ by 2.2 . The remaining side $A_{r} A_{1}$ of $E_{k}\left(\pi_{n}\right)$ is projected into the single vertex $\dot{A}_{r-1}$. Thus the projection of $E_{k}\left(\pi_{n}\right)$ from $A_{1}$ is the polygon $E_{k}\left(\pi_{n-1}\right)$ without the side $\dot{A}_{r-1} \dot{A}_{1}$.

Following the definition 4.1 the points $B_{2}^{k}, 1 \leqq k \leqq r-n-1$, are on the line $\left[A_{1}, A_{2}\right]$. We now prove that the points $A_{1}, A_{2}, B_{2}^{k-1}, B_{2}^{k}$ follow in order in the direction from $A_{1}$ to $A_{2}$ in the side $A_{1} A_{2}$ of $\pi_{n}$. If $1 \leqq k \leqq r-n-1$, none of the three vertices $A_{r}, A_{1}, A_{2}$ can occur in the $n-2$-space

$$
L=\left[A_{2+k}, A_{3+k}, \ldots, A_{n+k}\right]
$$

and so the polygon arc of all vertices not in $L$ has the form $A_{n+k+1}$ $A_{n+k+2} \ldots A_{r} A_{1} A_{2} \ldots A_{1+k}$. If $s$ is a point of this arc then $s \notin L$. This follows from 2.1 if $s$ is a vertex. It is also true if $s$ is not a vertex, for otherwise the space generated by $L$ and the two endpoints of the side containing $s$ would have at most dimension $n-1$ and contain $n+1$ vertices of $\pi_{n}$. This also proves that $[L, s]$ is a hyperplane which intersects $\pi_{n}$ in $s$. As $[L, s]$ intersects $\pi_{n}$ in the $n-1$ vertices in $L$, it follows from the order of $\pi_{n}$ that two different positions of $s$ on the polygon arc define different hyperplanes $[L, s]$. Hence, as $s$ runs continuously and monotonously through the polygon arc from $A_{n+k+1}$ through $A_{1}, A_{2}$ to $A_{1+k}$, $[L, s]$ moves continuously and monotonously within the hyperplane pencil through $L$. Therefore, as $[L, s]$ contains exactly one point of the line $\left[A_{1}, A_{2}\right]$, the intersection $\left[A_{1}, A_{2}\right] \cap[L, s]$ runs continuously and monotonously on $\left[A_{1}, A_{2}\right]$ from

$$
\left[A_{1}, A_{2}\right] \cap\left[A_{2+k}, A_{3+k}, \ldots, A_{n+k+1}\right]=B_{2}^{k}
$$

through $A_{1}$ and $A_{2}$ to

$$
\left[A_{1}, A_{2}\right] \cap\left[A_{1+k}, A_{2+k}, \ldots, A_{n+l}\right]=B_{2}^{k-1} .
$$

This proves that $A_{1}, A_{2}, B_{2}^{k-1}, B_{2}^{k}$ follow on $\left[A_{1}, A_{2}\right]$ in the order defined by the direction $A_{1}$ to $A_{2}$ in the side $A_{1} A_{2}$.

In particular the points $A_{1}, A_{2}, B_{2}^{1}$ follow in the order $A_{1}$ to $A_{2}$ on $A_{1} A_{2}$. By 4.3 the side $B_{1}^{1} B_{2}^{1}=A_{1} B_{2}^{1}$ of $E_{1}\left(\pi_{n}\right)$ is defined to contain $B_{2}^{0}=A_{2}$ as an interior point. Therefore the segment $B_{1}^{1} B_{2}^{1}$ contains the side $B_{1}^{0} B_{2}^{0}=A_{1} A_{2}$ of $E_{0}\left(\pi_{n}\right)=\pi_{n}$ as a subsegment. More generally as, $A_{1}, A_{2}$, 
$B_{2}^{k-1}, B_{2}^{k}$ follow in the order $A_{1}$ to $A_{2}$ and $B_{2}^{k-1}$ is an interior point of the side $B_{1}^{k} B_{2}^{k}$ of $E_{k}\left(\pi_{n}\right)$ it follows that $B_{1}^{k} B_{2}^{k}$ contains the side $B_{1}^{k-1} B_{2}^{k-1}$ of $E_{k-1}\left(\pi_{n}\right)$ as a subsegment, $1 \leqq k \leqq r-n-1$. If this result is applied to $\pi_{n-1}$ it follows that the side $\dot{B}_{1}^{k} \dot{B}_{2}^{k}$ of $E_{k}\left(\pi_{n-1}\right)$ contains the side $\dot{B}_{1}^{k-1} \dot{B}_{2}^{k-1}$ of $E_{k-1}\left(\pi_{n-1}\right)$ as a subsegment.

By the result of the first paragraph the projection of the segment $B_{2}^{k} B_{3}^{k-1}$ of the side $B_{2}^{k} B_{3}^{k}$ of $E_{k}\left(\pi_{n}\right)$ is the segment of the side $\dot{B}_{1}^{k} \dot{B}_{2}^{k}$ of $E_{k}\left(\pi_{n-1}\right)$ with endpoints $\dot{B}_{1}^{k}=\dot{A}_{1}=\dot{B}_{1}^{k-1}$ and $\dot{B}_{2}^{k-1}$. This by the paragraph above is the side $\dot{B}_{1}^{k-1} \dot{B}_{2}^{k-1}$ of $E_{k-1}\left(\pi_{n-1}\right)$. As the projection of the side $B_{2}^{k-1} B_{3}^{k-1}$ of $t_{2}^{k}$ is also $\dot{B}_{1}^{k-1} \dot{B}_{2}^{k-1}$ it follows that $t_{2}^{k}$ is projected into this single segment. The centre of projection $A_{1}$ is not located on a side of $t_{2}^{k}$ as it is on the line $\left[A_{1}, A_{2}\right]$ containing the side $B_{2}^{k-1} B_{2}^{k}$ but not within $B_{2}^{k-1} B_{2}^{k}$. Therefore $t_{2}^{k}$ is even because the projection of an odd triangle from a point not on the triangle is the full projective line. In particular this proves the result for $n=2$.

We assume it true for all polygons $\pi_{n-1}, n>2$, and proceed by induction. We need now only consider triangles $t_{i}^{k}$ with $i>2$. Let $B_{i}^{k-1} B_{i+1}^{k-1}$, $B_{i}^{k-1} B_{i}^{k}, B_{i}^{k} B_{i+1}^{k-1}$ be the sides of such a triangle $t_{i}^{k}$. Then by the result of the first paragraph the projection of $t_{i}^{k}$ is a triangle $\dot{t}_{i}^{k}$ defined for the projection $E_{k}\left(\pi_{n-1}\right)$. By the induction assumption $t_{i}^{k}$ is even, hence $t_{i}^{k}$ is even. This completes the proof.

$4.5 E_{r-n-1}\left(\pi_{n}\right)$ is not within a hyperplane, has $n+1$ vertices and order $n$.

Proof. We first show that

$$
\left[B_{n+1-j}^{k}, B_{n+2-j}^{k}, \ldots, B_{n+1}^{k}\right]=\left[A_{n+k+1-j}, A_{n+k+2-j}, \ldots, A_{n+k+1}\right],
$$

$0 \leqq k \leqq r-n-1,0 \leqq j \leqq n-1$. For $j=0$ this is an immediate consequence of 4.2. We assume the result to be true for $j-1, j>0$, and proceed by induction. According to the induction assumption

$$
\left[B_{n+2-j}^{k}, B_{n+3-j}^{k}, \ldots, B_{n+1}^{k}\right]=\left[A_{n+k+2-j}, A_{n+k+3-j}, \ldots, A_{n+k+1}\right] .
$$

It follows from the definition 4.1 that

and so

$$
B_{n+1-j}^{k} \in\left[A_{n+k+1-j}, A_{n+k+2-j}, \ldots, A_{n+k+1}\right]
$$

$$
\left[B_{n+1-j}^{k}, B_{n+2-j}^{k}, \ldots, B_{n+1}^{k}\right] \subseteq\left[A_{n+k+1-j}, A_{n+k+2-j}, \ldots, A_{n+k+1}\right] .
$$

\section{However}

$$
B_{n+1-j}^{k} \notin\left[A_{n+k+2-j}, A_{n+k+3-j}, \ldots, A_{n+k+1}\right] .
$$

For otherwise, as by $4.1 B_{n+1-j}^{k} \in\left[A_{1}, A_{2}, \ldots, A_{n+1-j}\right]$, the intersection

$$
\left[A_{1}, A_{2}, \ldots, A_{n+1-j}\right] \cap\left[A_{n+k+2-j}, A_{n+k+3-j}, \ldots, A_{n+k+1}\right]
$$


would not be empty. It follows from 2.1 that this intersection is an intersection of spaces of dimension $n-j$ and $j-1$ which together generate the projective $n$-space. Hence, if $d$ is the dimension of the intersection, it follows from the incidence relation $(n-j)+(j-1)=n-d$ that $d=-1$ which means that the intersection is empty. This contradiction proves

$$
B_{n+1-j} \notin\left[A_{n+k+2-j}, A_{n+k+3-j}, \ldots, A_{n+k+1}\right] .
$$

It now follows that

$$
\left[B_{n+1-j}^{k}, B_{n+2-j}^{k}, \ldots, B_{n+1}^{k}\right]=\left[A_{n+k+1-j}, A_{n+k+2-j}, \ldots, A_{n+k+1}\right]
$$

as the left hand side is included in the right hand side and both sides have the same dimension. We now specialize $j$ to be $n-1$. As in 4.1 we assume $r>n+1$ and so $r-n-2 \geqq 0$. We may therefore specialize $k$ to be $r-n-2$. The result becomes

$$
\left[B_{2}^{r-n-2}, B_{3}^{r-n-2}, \ldots, B_{n+1}^{r-n-2}\right]=\left[A_{r-n}, A_{r-n+1}, \ldots, A_{r-1}\right] .
$$

The vertices of $E_{n-r-1}\left(\pi_{n}\right)$ are defined to be $B_{1}^{r-n-1}\left(=A_{1}\right), B_{2}^{n-r-1}, \ldots$, $B_{n+1}^{r-n-1}\left(=A_{r}\right)$. Following the definition 4.3 each side $B_{i}^{r-n-1} B_{i+1}^{r-n-1}$ contains the interior point $B_{i+1}^{r-n-2}, 1 \leqq i \leqq n$. By the previous paragraph,

$$
\left[B_{2}^{r-n-2}, B_{3}^{r-n-2}, \ldots, B_{n+1}^{r-n-2}\right]=\left[A_{r-n}, A_{r-n+1}, \ldots, A_{r-1}\right] .
$$

It follows, then, that the $n+1$ vertices of $E_{r-n-1}\left(\pi_{n}\right)$ generate the space $\left[A_{r-n}, A_{r-n+1}, \ldots, A_{r}\right]$, that is, the full projective $n$-space. Consequently $E_{r-n-1}\left(\pi_{n}\right)$ cannot be included in a hyperplane. Moreover the hyperplane $\left[B_{2}^{r-n-2}, B_{3}^{r-n-2}, \ldots, B_{n+1}^{r-n-2}\right]$ generated by $n$ points, one interior to each of $n$ consecutive sides of $E_{r-n-1}\left(\pi_{n}\right)$, cannot intersect the side $A_{r} A_{1}$ of $E_{r-n-1}\left(\pi_{n}\right)$ as it already intersects $\pi_{n}$ in the $n$ vertices $A_{r-n}, A_{r-n-1}, \ldots$, $A_{r-1}$. It follows, then, from 2.3 that $E_{r-n-1}\left(\pi_{n}\right)$ has order $n$. This completes the proof.

4.6 For $n \geqq 2$ the polygon $E_{k}\left(\pi_{n}\right)$ has order $n . E_{k-1}\left(\pi_{n}\right)$ is inscribed in $E_{k}\left(\pi_{n}\right)$ in the sense of $3.1,1 \leqq k \leqq r-n-1$.

Proof. By $4.5 E_{r-n-1}\left(\pi_{n}\right)$ has order $n$. We assume $E_{k}\left(\pi_{n}\right)$ has order $n, 1<k \leqq r-n-1$, and proceed by induction. Each of the $n$ consecutive sides $B_{i}^{k} B_{i+1}^{k}, 1 \leqq i \leqq n$, of $E_{k}\left(\pi_{n}\right)$ contains the vertex $B_{i+1}^{k-1}$ of the polygon $E_{k-1}\left(\pi_{n}\right)$ in accordance with 4.3. By 4.4 the triangle $t_{i}^{k}$ which consists of the side $B_{i}^{k-1} B_{i+1}^{k-1}$ of $E_{k-1}\left(\pi_{n}\right)$ and the segments $B_{i}^{k-1} B_{i}^{k}, B_{i}^{k} B_{i+1}^{k-1}$ of the sides $B_{i-1}^{k} B_{i}^{k}, B_{i}^{k} B_{i+1}^{k}$ of $E_{k}\left(\pi_{n}\right)$, respectively, is even, $2 \leqq i \leqq n$. This means that the polygon $E_{k-1}\left(\pi_{n}\right)$ which is defined to have the sides $B_{1}^{k-1} B_{2}^{k-1}\left(=A_{1} B_{2}^{k-1}\right), \quad B_{2}^{k-1} B_{3}^{k-1}, \ldots, B_{n}^{k-1} B_{n+1}^{k-1}, B_{n+1}^{k-1} B_{n+1}^{k}\left(=A_{n+k} A_{n+k+1}\right)$, $A_{n+k+1} A_{n+k+2}, \ldots, A_{r} A_{1}$ is inscribed in $E_{k}\left(\pi_{n}\right)$ following 3.1 . By the in- 
duction assumption $E_{k}\left(\pi_{n}\right)$ has order $n$. Therefore by $3.2 E_{k-1}\left(\pi_{n}\right)$ has order $n$ and the proof is complete.

\section{The osculating hyperplanes of $\pi_{n}$.}

5.1 Definition. If, for $n \geqq 2, A_{i}, A_{i+1}, \ldots, A_{i+n}$ are vertices of a polygon $\pi_{n}$ and $A_{i+n} A_{i}$ is the straight line segment for which the sides $A_{i} A_{i+1}$, $A_{i+1} A_{i+2}, \ldots, A_{i+n-1} A_{i+n}$ of $\pi_{n}$ and $A_{i+n} A_{i}$ form a closed polygon of order $n$, then the hyperplanes which contain $A_{i+1}, A_{i+2}, \ldots, A_{i+n-1}$ but do not contain an interior point of $A_{i+n} A_{i}$ are defined to be osculating hyperplanes of $\pi_{n}$.

It follows from 2.3 that the segment $A_{i+n} A_{i}$ can always be constructed. This segment is unique.

If $\pi_{n}$ is closed, the hyperplanes

$$
\begin{array}{r}
V_{1}=\left[A_{1}, A_{2}, \ldots, A_{n}\right], V_{2}=\left[A_{2}, A_{3}, \ldots, A_{n+1}\right], \ldots, \\
V_{r}=\left[A_{r}, A_{1}, \ldots, A_{n-1}\right]
\end{array}
$$

satisfy the definition for the osculating hyperplanes. These hyperplanes will be called the vertex hyperplanes of $\pi_{n}$.

The osculating hyperplanes which contain

$$
V_{i} \cap V_{i+1}=\left[A_{i+1}, A_{i+2}, \ldots, A_{i+n-1}\right]
$$

and an interior point of the segment complementary to $A_{i+n} A_{i}$ form a linear segment of the hyperplane pencil through $\left[A_{i+1}, A_{i+2}, \ldots, A_{i+n-1}\right]$ bounded by the two hyperplanes $V_{i}, V_{i+1}$. The hyperplanes of this segment will be denoted by $V_{i} V_{i+1}$.

5.2 If, for $n \geqq 2, \pi_{n}$ is a closed polygon with vertices $A_{1}, A_{2}, \ldots, A_{r}$, $H$ is an osculating hyperplane interior to the segment $V_{1} V_{2}$ and $H^{\prime}$ is a hyperplane which approaches $H$ in such a way that it always contains a point interior to each side $A_{i} A_{i+1}, 1 \leqq i \leqq n-1$, then $H^{\prime}$ intersects $\pi_{n}$ in these points and also in an additional point interior to the side $A_{n} A_{n+1}$ providing it is sufficiently close to $H$.

Proof. Let $B_{i+1}$ be the point interior to $A_{i} A_{i+1}$ which is contained within $H^{\prime}, 1 \leqq i \leqq n-1$. It follows from the existence of these points that if $H^{\prime}$ contains one of the vertices $A_{1}, A_{2}, \ldots, A_{n}$ then it contains all of them and so becomes the hyperplane $V_{1}=\left[A_{1}, A_{2}, \ldots, A_{n}\right]$. By the hypothesis $H$ is interior to the segment $V_{1} V_{2}$ and so $H \neq V_{1}$. Hence if $H^{\prime}$ is sufficiently close to $H$ it cannot contain any vertex $A_{1}, A_{2}, \ldots, A_{n}$ and so it intersects $\pi_{n}$ in $B_{2}, B_{3}, \ldots, B_{n}$. If $A_{n+1} A_{1}$ be the segment 
defined in 5.1, let $-A_{n+1} A_{1}$ be the complement of $A_{n+1} A_{1}$ in the straight line $\left[A_{n+1}, A_{1}\right]$. As $H$ is interior to $V_{1} V_{2}, H$ intersects $-A_{n+1} A_{1}$ in an interior point and so $H^{\prime}$ intersects $-A_{n+1} A_{1}$ in an interior point if it is sufficiently close to $H$. If this position of $H^{\prime}$ did not intersect $A_{n} A_{n+1}$ then by 2.3 the polygon with sides $-A_{n+1} A_{1}, A_{1} A_{2}, \ldots, A_{n} A_{n+1}$ would have order $n$. This is impossible as $A_{n+1} A_{1}$ is defined in 5.1 so that $A_{n+1} A_{1}, A_{1} A_{2}, \ldots, A_{n} A_{n+1}$ has order $n$. This completes the proof.

5.3 If, for $n \geqq 2, H$ is a hyperplane interior to the segment $V_{i} V_{i+1}$ defined for a closed polygon $\pi_{n}$ with vertices $A_{1}, A_{2}, \ldots, A_{r}$, then the two intersections

$$
\pi_{n} \cap H, \quad \pi_{n} \cap V_{i} \cap V_{i+1},
$$

$1 \leqq i \leqq r$, are the same point set.

Proof. Without restriction in generality we may assume $i=1$ as, because $\pi_{n}$ is closed, the vertex notation can be adjusted so that $V_{i} V_{i+1}$ becomes $V_{1} V_{2}$. The vertex hyperplanes $V_{1}, V_{2}$ are defined, in 5.1 , to be $\left[A_{1}, A_{2}, \ldots, A_{n}\right]$ and $\left[A_{2}, A_{3}, \ldots, A_{n+1}\right]$ respectively. Therefore $V_{1} \cap V_{2}=$ $\left[A_{2}, A_{3}, \ldots, A_{n}\right]$. Consequently the set of points $\alpha$ of the polygon $\operatorname{arc} A_{2} A_{3} \ldots A_{n}$ of $\pi_{n}$ is contained within $V_{1} \cap V_{2}$. Hence, as $H$ is defined to contain $V_{1} \cap V_{2}$,

$$
\alpha \subseteq \pi_{n} \cap V_{1} \cap V_{2} \subseteq \pi_{n} \cap H .
$$

To prove the result it is therefore sufficient to show that

$$
\pi_{n} \cap H \subseteq \alpha .
$$

No point of the side $A_{1} A_{2}$ other than $A_{2}$ can be within $H$ for otherwise $H$ would be $\left[A_{1}, V_{1} \cap V_{2}\right]=V_{1}$ contrary to the assumption that $H$ is interior to the segment $V_{1} V_{2}$. Similarly no point of $A_{n} A_{n+1}$ other than $A_{n}$ can be within $H$. It remains to show that $H$ cannot contain any point $s$ interior to the polygon arc $A_{n+1} A_{n+2} \ldots A_{r} A_{1}$. Suppose such a point $s$ exists. Then $s \notin\left[A_{2}, A_{3}, \ldots, A_{n}\right]$. This is clear if $s$ is a vertex. If $s$ is interior to a side $A_{j} A_{j+1}$ of the arc containing $s$ then it is also true for otherwise $\left[A_{2}, A_{3}, \ldots, A_{n}, A_{j}, A_{j+1}\right]$ would have at most dimension $n-1$ and contain $n+1$ distinct vertices in contradiction to 2.1. It follows then that $H=\left[s, A_{2}, A_{3}, \ldots, A_{n}\right]$ and also that $H$ intersects $\pi_{n}$ in $s$. Let $B_{i+1}$ be a point interior to the side $A_{i} A_{i+1}, 1 \leqq i \leqq n-1$. If $B_{3}, B_{4}, \ldots, B_{n}$ remain fixed but $B_{2}$ approaches $A_{2}$ then $\left[B_{2}, B_{3}, \ldots, B_{n}\right]$ approaches

$$
\left[A_{2}, B_{3}, B_{4}, \ldots, B_{n}\right]=\left[A_{2}, A_{3}, \ldots, A_{n}\right] .
$$

Hence $\left[s, B_{2}, B_{3}, \ldots, B_{n}\right]$ approaches $H$ and, if $B_{2}$ is sufficiently close to $A_{2}$, is therefore a hyperplane which intersects $\pi_{n}$ in $s$. By 5.2 
$\left[s, B_{2}, B_{3}, \ldots, B_{n}\right]$, besides intersecting $\pi_{n}$ in $B_{2}, B_{3}, \ldots, B_{n}$, also intersects $\pi_{n}$ in a point $B_{n+1}$ interior to the side $A_{n} A_{n+1}$ again provided $B_{2}$ is sufficiently close to $A_{2}$. As $s \notin A_{n} A_{n+1},\left[s, B_{2}, B_{3}, \ldots, B_{n}\right]$ intersects $\pi_{n}$ in the $n+1$ distinct points $s, B_{2}, B_{3}, \ldots, B_{n+1}$ which is impossible because of the order of $\pi_{n}$. Hence $H$ cannot contain a point $s$ interior to the arc $A_{n+1} A_{n+2} \ldots A_{r} A_{1}$. Therefore $\pi_{n} \cap H$ is contained in the arc $\alpha$, and the result is proved.

5.4 If , for $n \geqq 2, A_{1}, A_{2}, \ldots, A_{r}$ are the vertices of a closed polygon $\pi_{n}$ and $B_{i+1} \in A_{i} A_{i+1}, 1 \leqq i \leqq n$ are vertices of a polygon $I\left(\pi_{n}\right)$ inscribed in $\pi_{n}$, then the vertex hyperplanes of $I\left(\pi_{n}\right)$ are the vertex hyperplanes of $\pi_{n}$ together with the hyperplane $\left[B_{2}, B_{3}, \ldots, B_{n+1}\right]$. All the osculating hyperplanes of $\pi_{n}$ except those interior to the segment $V_{1} V_{2}$ are likewise osculating hyperplanes of $I\left(\pi_{n}\right)$.

Proof. As $A_{1}, B_{2}, B_{3}, \ldots, B_{n+1}, A_{n+1}, \ldots, A_{r}$ are consecutive vertices of $I\left(\pi_{n}\right)$,

$$
\begin{aligned}
& {\left[A_{1}, B_{2}, \ldots, B_{n}\right],} \\
& {\left[B_{2}, B_{3}, \ldots, B_{n+1}\right],} \\
& {\left[B_{3}, B_{4}, \ldots, B_{n+1}, A_{n+1}\right],} \\
& \ldots \ldots \ldots . \ldots . \ldots . . . \\
& {\left[A_{r}, A_{1}, B_{2}, \ldots, B_{n-1}\right]}
\end{aligned}
$$

are the vertex hyperplanes of $I\left(\pi_{n}\right)$. As $B_{i+1}$ by its definition is an interior point of the segment $A_{i} A_{i+1}, 1 \leqq i \leqq n$, the above hyperplanes are, respectively,

$$
\begin{aligned}
& {\left[A_{1}, A_{2}, \ldots, A_{n}\right],} \\
& {\left[B_{2}, B_{3}, \ldots, B_{n+1}\right],} \\
& {\left[A_{2}, A_{3}, \ldots, A_{n+1}\right],} \\
& \ldots \ldots . \ldots . \ldots . . . \\
& {\left[A_{r}, A_{1}, A_{2}, \ldots, A_{n-1}\right] .}
\end{aligned}
$$

These are exactly the vertex hyperplanes of $\pi_{n}$ together with the additional hyperplane $\left[B_{2}, B_{3}, \ldots, B_{n}\right]$. Thus the first part of the result is established.

This means, for $2 \leqq i \leqq r$, that $V_{i}, V_{i+1}$ besides being consecutive vertex hyperplanes of $\pi_{n}$ are also consecutive vertex hyperplanes of $I\left(\pi_{n}\right)$. By 5.1 $V_{i} V_{i+1}$ is one of the two segments of the hyperplane pencil through $V_{i} \cap V_{i+1}$ bounded by $V_{i}$ and $V_{i+1}$. To prove that $V_{i} V_{i+1}$ is the same whether it is defined for $\pi_{n}$ or $I\left(\pi_{n}\right)$ it is sufficient to show a hyperplane $H$ exists in the pencil which is different from $V_{i}$ and $V_{i+1}$ and is neither an osculating hyperplane of $\pi_{n}$ nor of $I\left(\pi_{n}\right)$. If the side $A_{i+n} A_{i+n+1}$ is also 
a side of $I\left(\pi_{n}\right)$ let $s$ be any interior point of this side. If $A_{i+n} A_{i+n+1}$ is not a side of $I\left(\pi_{n}\right)$ then the vertex $B_{i+n+1}$ is an interior point of this side. In this case $s$ is defined to be $B_{i+n+1}$.

$$
s \notin\left[A_{i+1}, A_{i+2}, \ldots, A_{i+n-1}\right]=V_{i} \cap V_{i+1}
$$

for otherwise $\left[A_{i+1}, A_{i+2}, \ldots, A_{i+n+1}\right]$ would have dimension at most $n-1$ and contain $n+1$ vertices of $\pi_{n}$ in contradiction to 2.1. Therefore $\left[s, A_{i+1}, A_{i+2}, \ldots, A_{i+n-1}\right]=H$ is a hyperplane. As $H$ intersects $A_{i+n} A_{i+n+1}$ in the interior points $s, H \neq V_{i}, H \neq V_{i+1}$. As $s \notin V_{i} \cap V_{i+1}, s \in \pi_{n}, s \in I\left(\pi_{n}\right)$, it follows from 5.3 that $H$ is not an osculating hyperplane of either $\pi_{n}$ or $I\left(\pi_{n}\right)$. This proves that $V_{i} V_{i+1}, 2 \leqq i \leqq r$, is the same whether it is defined for $\pi_{n}$ or $I\left(\pi_{n}\right)$ as its complementary segment consists of hyperplanes which are not osculating hyperplanes of either $\pi_{n}$ or $I\left(\pi_{n}\right)$. The proof is now complete.

5.5 No space point is within more than $n$ vertex hyperplanes of a polygon $\pi_{n}$.

Proof. As the result is trivial for $n=1$ we assume $n \geqq 2$. Let $A_{1}, A_{2}, \ldots, A_{r}$ be the vertices of $\pi_{n}$. If $r$ assumes its minimum value $n+1$ then, as $\pi_{n}$ satisfies the dimension condition, $A_{1}, A_{2}, \ldots, A_{n+1}$ are the vertices of an $n$-simplex while the vertex hyperplanes

$$
\begin{aligned}
& V_{1}=\left[A_{1}, A_{2}, \ldots, A_{n}\right] \\
& V_{2}=\left[A_{2}, A_{n}, \ldots, A_{n+1}\right] \\
& \ldots \ldots \ldots . . . . . . . \\
& V_{n+1}=\left[A_{n+1}, A_{1}, \ldots, A_{n-1}\right]
\end{aligned}
$$

of $\pi_{n}$ are the face hyperplanes of this $n$-simplex. As no point can be within all the face hyperplanes of a simplex no point can be within the $n+1$ vertex hyperplanes of $\pi_{n}$. This proves the result if $r=n+1$. We assume it is true for all polygons $\pi_{n}$ with $r-1$ sides, $r>n+1$, and proceed by induction.

Now let $Q$ be a given space point. As $r>n+1, E_{1}\left(\pi_{n}\right)$ is defined as in 4.3. By 4.6 $E_{1}\left(\pi_{n}\right)$ has order $n$ while $E_{0}\left(\pi_{n}\right)$, i.e. $\pi_{n}$, is inscribed in $E_{1}\left(\pi_{n}\right)$. As $E_{1}\left(\pi_{n}\right)$ has one vertex less than $\pi_{n}$ the induction assumption may be applied to it. Hence $Q$ is included in at most $n$ vertex hyperplanes of $E_{1}\left(\pi_{n}\right)$. As $E_{1}\left(\pi_{n}\right)$ has at least $n+1$ vertices this means that at least one vertex hyperplane of $E_{1}\left(\pi_{n}\right)$ exists which does not contain $Q$. By 5.4 each vertex hyperplane of $E_{1}\left(\pi_{n}\right)$ is also a vertex hyperplane of $\pi_{n}$. Hence at least one vertex hyperplane of $\pi_{n}$ exists which does not contain $Q$. By adjusting the vertex notation, if necessary, it may be assumed that 
$\left[A_{2}, A_{3}, \ldots, A_{n+1}\right]$ is such a vertex hyperplane. As $A_{i+1}$ is an interior point of the side $B_{i}^{1} B_{i+1}^{1}, 1 \leqq i \leqq n$, of $E_{1}\left(\pi_{n}\right)$ it follows from 5.4 that the vertex hyperplanes of the polygon $\pi_{n}$ inscribed in $E_{1}\left(\pi_{n}\right)$ are the vertex hyperplanes of $E_{1}\left(\pi_{n}\right)$ together with $\left[A_{2}, A_{3}, \ldots, A_{n+1}\right]$. By the induction assumption $Q$ is contained within at most $n$ vertex hyperplanes of $E_{1}\left(\pi_{n}\right)$. As $Q \notin\left[A_{2}, A_{3}, \ldots, A_{n+1}\right], Q$ is within at most $n$ vertex hyperplanes of $\pi_{n}$. This completes the proof.

5.6 (The DUality theorem). If, for $n \geqq 2, V_{1}, V_{2}, \ldots, V_{r}$ are the vertex hyperplanes of a closed polygon $\pi_{n}$ then the dual of the system $\Pi$ of all the hyperplanes of the segments $V_{1} V_{2}, V_{2} V_{3}, \ldots, V_{r} V_{1}$ is also a closed polygon of order $n$ which satisfies the dimension condition.

Proof. It follows from 5.5 that at most $n$ of the hyperplanes $V_{1}, V_{2}, \ldots, V_{r}$ of $\pi_{n}$ can pass through a given point. Because $\pi_{n}$ satisfies the dimension condition, $r>n$ and so not all of the hyperplanes of $\Pi$ can pass through a given point. Therefore the dual of $\Pi$ satisfies the dimension condition.

To show that the dual of the system $\Pi$ has order $n$ we must show, in accordance with the definition of an intersection point in 1 , that a given space point $Q$ is contained in at most $n$ hyperplanes which belong to either of the following two types. The first type consists of the vertex hyperplanes of $\pi_{n}$ while the second type consists of hyperplanes of the segments $V_{i} V_{i+1}$ for which $Q \notin V_{i}, Q \notin V_{i+1}$. This is done by constructing a polygon $\bar{\pi}_{n}$ of order $n$ so that there is a one to one correspondence between the hyperplanes of the above two types which contain $Q$ and vertex hyperplanes of $\bar{\pi}_{n}$ which contain $Q$. By applying 5.5 to $\bar{\pi}_{n}$ it follows that there are at most $n$ vertex hyperplanes of $\bar{\pi}_{n}$ which contain $Q$. Hence there are at most $n$ hyperplanes of the above two types which contain $Q$.

To complete the proof it only remains to construct a polygon $\bar{\pi}_{n}$. If no hyperplanes of the second type contain $Q$ then $\bar{\pi}_{n}$ is defined to be $\pi_{n}$. Suppose then that a hyperplane $H$ of the second type contains $Q$. The vertex notation may be adjusted so that $H$ belongs to the segment $V_{1} V_{2}$. As $H$ is of the second type $Q \notin V_{1} \cap V_{2}$, and so $H=\left[V_{1} \cap V_{2}, Q\right]$. Now let $B_{i+1}$ be a point in the interior of the side $A_{i} A_{i+1}, 1 \leqq i \leqq n-1$. If $B_{3}, B_{4}, \ldots, B_{n}$ remain fixed but $B_{2}$ approaches $A_{2}$ then $\left[B_{2}, B_{3}, \ldots, B_{n}, Q\right]$ approaches

$$
\left[A_{2}, B_{3}, \ldots, B_{n}, Q\right]=\left[A_{2}, A_{3}, \ldots, A_{n}, Q\right]=\left[V_{1} \cap V_{2}, Q\right]=H .
$$

Therefore by 5.2 if $B_{2}$ is sufficiently close to $A_{2}$ then $\left[B_{2}, B_{3}, \ldots, B_{n}, Q\right]$ is a hyperplane which intersects $\pi_{n}$ in a point $B_{n+1}$ interior to $A_{n} A_{n+1}$ 
as well as in $B_{2}, B_{3}, \ldots, B_{n}$. Let $I\left(\pi_{n}\right)$ be the polygon inscribed in $\pi_{n}$ defined as in 3.1 for the points $B_{2}, B_{3}, \ldots, B_{n+1}$. By $3.2 I\left(\pi_{n}\right)$ has order $n$ and so $\left[B_{2}, B_{3}, \ldots, B_{n+1}\right]$ is a hyperplane. Therefore $Q \in\left[B_{2}, B_{3}, \ldots, B_{n+1}\right]$. This hyperplane cannot be an osculating hyperplane of $\pi_{n}$ as such hyperplanes intersect $\pi_{n}$ only in vertices. All the osculating hyperplanes of $\pi_{n}$ which do not belong to the segment $V_{1} V_{2}$ are osculating hyperplanes of $I\left(\pi_{n}\right)$ by 5.4, and in particular a vertex hyperplane of $\pi_{n}$ is likewise a vertex hyperplane of $I\left(\pi_{n}\right)$. We define $H$ to correspond to the vertex hyperplane $\left[B_{2}, B_{3}, \ldots, B_{n+1}\right]$ of $I\left(\pi_{n}\right)$ and each of the other osculating hyperplanes of $\pi_{n}$ which contains $Q$ to correspond to the same osculating hyperplane of $I\left(\pi_{n}\right)$. As $\left[B_{2}, B_{3}, \ldots, B_{n+1}\right]$ cannot be an osculating hyperplane of $\pi_{n}$ this is a one to one correspondence. Moreover each vertex hyperplane corresponds to a vertex hyperplane while one of the hyperplanes of the second type, namely $H$, corresponds to a vertex hyperplane. By $3.2 I\left(\pi_{n}\right)$ has order $n$. Hence the above process may in turn be applied to $I\left(\pi_{n}\right)$ and then repeated until all the hyperplanes of the two types which contain $Q$ are in a one to one correspondence with vertex hyperplanes of a polygon $\bar{\pi}_{n}$ of order $n$. With the construction of $\bar{\pi}_{n}$ the proof is complete.

\section{An application to curves of order $n$.}

The symbol $C_{n}$ is used in this section to represent a curve in real projective $n$-space which is homeomorphic to a circle of circumference of length 1 such that no hyperplane contains more than $n$ points of $C_{n}$. Let $Q$ be a point of the circumference. The distance $s$ measured along the circumference from a fixed point in a fixed direction to $Q$ determines $Q$ and so also the homeomorphic image of $Q$ on $C_{n}$. Thus $s$ serves as a coordinate to define the points of $C_{n}$. Accordingly the numbers $s$, com puted modulo 1, will be used to designate the points of $C_{n}$.

6.1 If $s_{1}, s_{2}, \ldots, s_{n q}$ represent points on a curve $C_{n}$ for which $0 \leqq s_{1}<s_{2}<\ldots<s_{n q}<1$, and if the spaces

$$
\left[s_{1}, s_{2}, \ldots, s_{n}\right], \ldots,\left[s_{n(q-1)+1}, s_{n(q-1)+2}, \ldots, s_{n q}\right]
$$

all pass through the same space point $Q$, then $q \leqq n$.

Proof. If $\bar{s}_{1}, \bar{s}_{2}, \ldots, \bar{s}_{n}$ are distinct points of $C_{n}$ then it follows from the order of $C_{n}$ that $\left[\bar{s}_{1}, \bar{s}_{2}, \ldots, \bar{s}_{n}\right]$ is a hyperplane and moreover $\bar{s}_{1}, \bar{s}_{2}, \ldots, \bar{s}_{n}$ are the only points of $C_{n}$ in this hyperplane. We now inscribe a polygon of order $n$ in $C_{n}$ with vertices $s_{1}, s_{2}, \ldots, s_{n q}$. We may assume $q>1$ for otherwise the result is trivial. It is convenient to define 
$s_{n q+1}=s_{1}$. Each arc of points $s$ with $s_{i} \leqq s \leqq s_{i+1} 1 \leqq i \leqq n q$, can be realized in an affine space obtained by removing the hyperplane generated by $n$ distinct points from the arc complementary to the arc $s_{i} \leqq s \leqq s_{i+1}$ from the projective space. We define the line segment $s_{i} s_{i+1}$ to be the straight line segment joining $s_{i}$ and $s_{i+1}$ in this affine space. $\pi$ is now defined to be the closed polygon with sides $s_{i} s_{i+1}, 1 \leqq i \leqq n q$. As $q>1$ this polygon satisfies the dimension condition. To prove it has order $n$ let $H$ be any hyperplane of the original projective $n$-space. If $H$ intersects $\pi$ in a vertex $s_{j}$ this vertex is also a point of $C_{n}$. If it intersects $\pi$ in an interior point of side $s_{i} s_{i+1}$ then as this side together with the corresponding $\operatorname{arc} s_{i}<s<s_{i+1}$ form a closed continuous curve within an affine space, $H$ must intersect the arc $s_{i}<s<s_{i+1}$ at least once. Hence the number of intersection points of $H$ and $\pi$ cannot exceed the number of intersection points of $H$ and $C_{n}$. As $C_{n}$ has order $n$ this proves that $\pi$ has order $n$.

The $q$ hyperplanes

$$
\left[s_{1}, s_{2}, \ldots, s_{n}\right],\left[s_{n+1}, s_{n+2}, \ldots, s_{2 n}\right], \ldots,\left[s_{n(q-1)+1}, s_{n(q-1)+2}, \ldots, s_{n q}\right]
$$

are all vertex hyperplanes of $\pi$. If these all pass through a point $Q$, then by 5.5 there are at most $n$ of them. Thus the result is proved.

\section{REFERENCES}

1. J. Hjelmslev, Om Polygoner og Polyedre, Mat. Tidsskr. B (1925), 65-73.

2. C. Juel, Einleitung in die Theorie der ebenen Elementarkurven dritter und vierter Ordnung, Danske Vid. Selsk. Skrifter (7) 2 (1914), 113-168.

3. E. Kivikoski, Uber Streckenzüge in der projektiven Ebene, Ann. Acad. Sci. Fennicae, Ser. A, 28, No. 14 (1928).

4. E. Kivikoski, Zur Theorie der projektiven Vielseite, Ann. Acad. Sci. Fennicae, Ser. A, 32, No. 3 (1929).

5. A. Marchaud, Sur les continus d'ordre borné, Acta Math. 55 (1930), 67-115.

6. P. Scherk, Über differenzierbare Kurven und Bögen II, Časopis Pěst. Mat. Fys. 66 (1937), 172-191. 